\title{
Indicadores para el control de la gestión de personal
}

Xavier Ballart * y Carles Ramió *

\section{Introducción}

Durante los últimos diez años, tanto en el sector público como en el privado, se han producido algunos intentos de llevar la función de Dirección y Gestión de Recursos Humanos más allá de la tradicional Dirección de Personal. Esta ha sido una oportunidad para que los recursos humanos adquirieran mayor importancia para el resto de la organización en sus programas de cambio. La literatura sobre sector privado ha destacado la necesidad de contar con el factor humano para que los planes estratégicos tuvieran éxito, dentro de un contexto en que los directores de recursos humanos muy a menudo habían quedado excluidos de la estrategegia de los procesos de cambio al considerarse que no aportaban conocimientos relevantes a los mismos (LaWLer, 1988; MILES y SNOw, 1984; SChuler y MacMillan, 1984; SCHuler, 1988). La literatura sobre el sector público también ha señalado la importancia de los recursos humanos en determinar estrategias de cambio factibles dada la naturaleza intensiva en personal de las actividades públicas, donde los recursos humanos suelen ser el recurso crítico, y donde el producto final son, cada vez más, servicios complejos e intensivos en conocimientos (REICH, 1983; MCGregor, 1988; Perry, 1993; Mesch, Perry y ReCansino Wise, 1995).

Existen diferentes aproximaciones a la gestión estratégica con respecto a la dirección de recursos humanos. La idea básica es, sin embargo, entrelazar la dirección del personal con el plan estratégico de la organización, mediante el ciclo de la Dirección y Gestión de Recursos Humanos. Esto significa gestionar el colectivo humano - selección, retribución, promoción, formación, régimen disciplinario, relaciones laborales- de modo que se satisfagan las demandas de la organización dentro de las condicionamientos que imponen las obligaciones legales y financieras. Todo ello implica un sistema integrado -las formas de retribuir al personal tienen que estar en con- sonancia con la evaluación del rendimiento, el tipo de selección, el plan de formación, etc...-, y éstos a su vez han de concordar con el conjunto de la estrategia de la organización (LAWLER, 1988).

Sin embargo, este modelo estratégico no es fácil de llevar a cabo. Las empresas privadas y las administraciones públicas sufren dificultades parecidas no sólo para fusionar la estrategia corporativa y las prácticas de Dirección y Gestión de Recursos Humanos sino también al intentar implantar una dirección estratégica de los recursos humanos. Algunos de los problemas básicos que afectan a la Dirección de Recursos Humanos todavía están relacionados, como sugieren los defensores de la gestión estratégica, con la naturaleza staff de los departamentos de personal, la separación entre la dirección del personal y la dirección efectiva de la organización —donde uno proporciona personal y el otro diseña los puestos de trabajo y planifica tareas y actividades- y la dispersión interna presente en los departamentos de personal, dada la variedad de especialidades y la tendencia a seguir cada una de ellas sus propios criterios.

Más específico del sector público es el papel marginal de los responsables de recursos humanos en el juego político interno de la administración, la codificación de las políticas de personal en leyes y reglamentos y la utilización de estructuras tradicionales, sobre la base de cátalogos de puestos de trabajo que inhiben la innovación y la flexibilidad. Además, generalmente en el sector público, es más difícil describir operativamente ula estrategia corporativa. (MOORE, 1995).

Este artículo se basa en la apreciación de que dar un paso adelante en la dirección estratégica de recursos humanos en el sector público requiere, por parte de los departamentos de personal, una mejor comprensión de las alternativas que pueden efectivamente perseguir, dados los recursos de que disponen, y de su rendimiento actual en cada una de las áreas que gestionan. Un primer paso es, por lo tanto, desarrollar un sistema de 
información con indicadores y medidas que ayude a los directivos de la Administración pública a desarrollar sus propias estrategias sobre la base de conocer con relativa prontitud y fiabilidad, si sus tentativas de actuación tienen éxito y, por tanto, si están consiguiendo sus propósitos.

En un proyecto de investigación que duró un año, realizado a petición de la Dirección General de la Función Pública de la Generalidad de Cataluña ', los autores ${ }^{2}$ idearon un conjunto de medidas que proporciona a los responsables del diseño de las políticas de personal una rápida visión de algunas áreas clave de la estructura y de las características de sus recursos humanos. En este artículo se presenta este sistema de indicadores. El artículo se ha estructurado en ocho apartados: el primer apartado presenta la lógica y los objetivos básicos del sistema. Los apartados restantes se corresponden con las siete áreas fundamentales que configuran las políticas de personal de la Administración analizada (características generales de los empleados públicos y la estructura de dirección; pautas de crecimiento y transformación; absentismo; selección, movilidad interna y promoción; retribución y evaluación del rendimiento; formación y socialización; y relaciones laborales).

\section{El sistema: factores críticos, múltiples indicadores, amplio pero simple y comprensible}

El sistema se concentra en los factores críticos, incluye indicadores múltiples sobre diferentes aspectos relacionados con el rendimiento de la gestión de personal y, al hacer esto, intenta ser amplio pero al mismo tiempo sencillo ya que su diseño debe facilitar al máximo su aplicación práctica. La idea de seleccionar un número reducido de factores críticos fue inicialmente reforzada por el deseo de los responsables de la Dirección General de la Función Pública en dirigir la atención de los distintos cargos directivos de la Administración de la Generalidad a una lista relativamente breve sobre la situación, la estructura y las tendencias de sus empleados públicos. Este era un modo de hacer converger las políticas de la Dirección General de la Función Pública con los planes de las Consejerías y ser aquella reconocida en el juego interno político-administrativo como un actor clave. Al mismo tiempo, también era un modo de traducir la misión general de la Dirección General de la Función Pública a unos objetivos concretos que son importantes para las Consejerías. Estas son los "Clientes" de la Dirección General de la Función Pública y aportarles valor pasó a ser la prioridad. Desde esta perspectiva, el sistema debía dar respuestas a pre- guntas que eran importantes para aquellas instancias que recibían los servicios.

La "perspectiva de los clientes" se equilibró con otras dos perspectivas: existía una perspectiva interna y una perspectiva más general sobre el coste-eficacia. La perspectiva interna estaba más enfocada hacia qué actividades la Dirección General de la Función Pública debía estar desarrollando y cómo debían llevarse a cabo para mejorar y para entrar en una estrategia de calidad. La perspectiva general de coste-eficacia era la más importante para algunos políticos y había sido formulada explícitamente en términos de reducir el número de los empleados públicos en un $2 \%$ en toda la Administración de la Generalidad en el plazo de un año.

Mientras existía un interés por comprobar el rendimiento en las distintas áreas de gestión de personal, se hizo un esfuerzo por minimizar la sobrecarga de información al limitar el número de indicadores. Una vez iniciado el proyecto y que los investigadores se habían familiarizado con la base de datos del personal de la Generalidad (Gestión Integrada del Personal GIP-) y con los procedimientos detallados de la administración de personal, era muy sencillo continuar anadiendo nuevos indicadores. Reuniones con los altos cargos de la Dirección General de la Función Pública, así como el contacto diario con los técnicos superiores de esta unidad, constituyeron una excelente fuente de nuevos indicadores corriéndose el riesgo de sustituir la calidad por la cantidad. Sin embargo, el objetivo básico y el valor añadido del sistema a implantar era conjugar diversas actividades aparentemente diferentes en un único, rápido, amplio y equilibrado informe.

Por otro lado, existía también el riesgo de no encontrar la información correcta debido a las limitaciones de la base de datos del personal o, también, por la disparidad en las fórmulas de computar los datos por parte de los servicios de personal de las distintas Consejerías. Desde esta perspectiva, el sistema se percibía como un modo de clarificar la visión de la Dirección General de la Función Pública y de ajustar progresivamente las prácticas en la gestión del personal a las políticas originarias. En el fondo, la cuestión principal era como inducir determinados comportamientos en las Consejerías en temas relacionados con personal. El sistema de indicadores apareció como una manera no sólo de proporcionar información sino también de difundir la experiencia y el aprendizaje de la Dirección General de la Función Pública a toda la Administración de la Generalidad. En la práctica, la mayoría de los responsables de los servicios de personal de cada una de las 14 Consejerías aceptó cooperar en el desarrollo del sistema ya que rápidamente percibieron los beneficios de tener datos con los que comparar su rendimiento con el resto de las Consejerías, siempre que los investigadores y la Dirección General de la Función Pública mantuvieran esta información dentro de la estricta confidencialidad. 


\section{Factores críticos e indicadores propuestos para su medida}

\section{CARACTERISTICAS GENERALES DE LOS EMPLEADOS PÚBLICOS Y DE LA ESTRUCTURA DE LAS JEFATURAS ADMINISTRATIVAS}

Uno de los mayores cambios a afrontar por la función de personal deriva de las transformaciones en la naturaleza del trabajo a desarrollar. Se producen diversos cambios simultáneamente. Por un lado, hay una transformación fundamental del trabajo operativo hacia el trabajo especializado que requiere más técnicos y funcionarios públicos muy profesionalizados (MCGREGOR, 1988). La complejidad jurídica en algunos temas y la complejidad política de otros a raíz de la apertura a los grupos de interés refuerzan estas tendencias. Por otro lado, la automatización de las tareas en las oficinas burocráticas, el aumento de la participación de los consumidores en la producción de servicios y, otras transformaciones en la interacción entre la administración y los ciudadanos están variando los conocimientos, las técnicas y las habilidades (KSAs: Knowledge, Skills y Abilities) que se exigen a los empleados públicos de primera fila o de contacto. Se han producido también cambios profundos en la gestión pública, tales como la contratación externa del suministro de servicios que se justifica en el deseo de reducir los gastos generales y de eliminar estructuras burocráticas sustituyéndolas por estructuras más planas, pasando la responsabilidad y la autonomía para actuar hacia abajo. Todo ello podría tener un mayor impacto en los recursos humanos de las administraciones públicas en la medida que estas transformaciones se vayan generalizando (DonaHuE, 1989, Kelman, 1990, HoOd, 1991, Barzelay, 1992).

Un segundo cambio significativo surge de la necesidad de redefinir el vínculo de la contratación de los empleados públicos con la Administración. En los sistemas continentales, tales como el francés, el italiano o el español, las reformas en la Administración pública que vienen produciéndose desde el siglo pasado han mantenido el vínculo de funcionario para un amplio espectro de puestos dentro de la Administración. Sólo cuando no existen limitaciones legales que obliguen a convocar una plaza de funcionario, se contempla la posibilidad de contratar empleados basándose en la legislación laboral ordinaria. Ambos sistemas de contratación otorgan, en general, a los empleados públicos, el mismo tipo de seguridad en la contrata- ción, excepto si éstos son contratados temporalmente debido a necesidades coyunturales de aumento de trabajo o restricciones presupuestarias. Por lo tanto, los empleados públicos pueden pertenecer a cuatro categorías diferentes (funcionarios, funcionarios interinos, laborales indefinidos y laborales temporales) dependiendo de la combinación de los distintos derechos estatutarios así como de la duración del contrato.

Una de las elecciones más críticas para los departamentos de personal se refiere a si se deben ampliar o reducir el número de puestos de trabajo reservados a los funcionarios. En el contexto actual de reforma de la legislación laboral, los funcionarios están considerados, cada vez más, como un sector que goza de privilegios importantes más que como una garantía de objetividad e independencia. Otra cuestión no menos importante es si se debe cubrir los puestos vacantes con contratos temporales a corto plazo o con contratos estables a largo plazo. La enorme estabilidad de la Administración pública permite planificar a largo plazo, lo que permite obtener los beneficios derivados de la estabilidad en el empleo.

También es importante para los departamentos de personal que exista una diversidad y un equilibrio respecto a la edad y el sexo de los empleados públicos y, más en concreto, entre los cargos directivos. El caso de Cataluña es un ejemplo de una administración que podemos calificar como "joven" con directivos y empleados de base que son lo suficientemente jóvenes como para continuar trabajando durante otros 20 ó 30 años más. También existe un gran equilibrio general por razón del género aunque hay diferencias notables entre las distintas Consejerías (Consejerías muy masculinizadas frente a Consejerías claramente femeninas). Las mujeres, además, tienden a ocupar muchos menos puestos directivos que los hombres.

Desarrollar oportunidades de promoción para los jọvenes y conseguir una representación más adecuada para la mujer en los niveles más elevados de la administración son otros factores críticos que pueden impulsar el diseño e implantación de políticas específicas. Tal como Drucker sugiere (1986), uno se puede sentir satisfecho y realizado con el trabajo que realiza más que por una rápida promoción. .Escaleras paralelas" de avance y gratificación por las contribuciones individuales tanto a nivel técnico como profesional son otras opciones a tener presentes (SCHULER 1988).

El primer grupo de indicadores sobre los recursos humanos que se utilizan para recoger información según las características generales de los empleados públicos y la estructura de las jefaturas se resume en el cuadro 1. En el cuadro no se incluyen los cruces posibles entre los diversos indicadores. 


\begin{tabular}{|c|c|}
\hline \multicolumn{2}{|c|}{$\begin{array}{l}\text { Cuadro } 1 \\
\text { Caracteristicas generales de los empleados públicos y } \\
\text { de la estructura de mandos por Consejerias }\end{array}$} \\
\hline Indicadores & Temas $R H$ \\
\hline $\begin{array}{l}\text { Empleos clasificados por grupos según } \\
\text { su nivel de estudios: } \\
A=\text { Título con } 4 \text { ó más anos en la Univ. } \\
B=\text { Título con } 2-3 \text { años en la Univ. } \\
C=B . U . P ., \text { Bachillerato } \\
D=\text { Educación General Básica } \\
E=\text { Certificado de Estudios }\end{array}$ & $\begin{array}{l}\text { Diferencias en los perfiles para uni- } \\
\text { dades similares. } \\
\text { Las posibilidades de promoción pue- } \\
\text { den condicionar la congelación en la } \\
\text { contratación de un grupo para per- } \\
\text { mitir la promoción interna. }\end{array}$ \\
\hline $\begin{array}{l}\text { Empleados por nivel de carrera y tra- } \\
\text { mos retributivos }(1-30)\end{array}$ & $\begin{array}{l}\text { Diferencias en los niveles de carreras } \\
\text { para trabajos similares. }\end{array}$ \\
\hline $\begin{array}{l}\text { Empleados en niveles inferiores con } \\
\text { carreras universitarias }\end{array}$ & $\begin{array}{l}\text { Fuentes potenciales de frustración. } \\
\text { Oportunidades para rediseñar los } \\
\text { puestos y para reformas en el sistema } \\
\text { retributivo. }\end{array}$ \\
\hline $\begin{array}{l}\text { Empleados por tipo de contrato: } \\
\text { - Funcionarios } \\
\text { - Interinos en la Admón. Pública } \\
\text { - Contrato laboral } \\
\text { - Contrato laboral temporal }\end{array}$ & $\begin{array}{l}\text { Derechos de los funcionarios vs. de- } \\
\text { rechos de los trabajadores. } \\
\text { Puestos estables-fijos vs. puestos ines- } \\
\text { tables-temporales. }\end{array}$ \\
\hline Porcentaje jefaturas/puestos base & $\begin{array}{l}\text { Estructura vertical vs. estructura pla- } \\
\text { na. }\end{array}$ \\
\hline $\begin{array}{l}\text { Empleados por edad y sexo en los } \\
\text { puestos de dirección y en otros pues- } \\
\text { tos }\end{array}$ & $\begin{array}{l}\text { Diferencias en las pirámides de edad } \\
\text { y sexo para la provisión de puesios } \\
\text { directivos y de puestos base. }\end{array}$ \\
\hline
\end{tabular}

Fuente: Elaboración propia.

\section{CRECIMIENTO Y PAUTAS DE CAMBIO}

Las presiones referentes a los costos no son nuevas en el sector público pero en la actualidad se están intensificado como consecuencia de la necesidad de reducir los déficit y de dar una respuesta a la preocupación pública sobre la relación costeeficacia. A las administraciones públicas se les requiere que frenen el crecimiento y que progresivamente reduzcan su tamaño congelando las contrataciones y facilitando incentivos a las salidas voluntarias de algunos de sus empleados. Para algunos responsables políticos y administrativos, el objetivo principal de los departamentos de personal es establecer controles estrictos en los gastos de personal y conseguir aumentar la productividad. En el caso de Cataluña, la voluntad de reducción del número de empleados públicos fue probablemente el objetivo operativo más claro de los dirigentes de la Dirección General de la Función Pública.

Políticas de congelación respecto a la contratación de nuevos trabajadores, los programas de jubilaciones anticipadas o las medidas que favorecen la marcha de los empleados públicos son las opciones básicas para mantener o reducir el volumen de personal. Las características generales de los emplea- dos que abandonan la Administración y los motivos de su marcha (cambio de personal, jubilación anticipada, permiso temporal, no renovación de los contratos temporales) dan una idea de qué elementos pueden producir un cambio más significativo en el conjunto de los efectivos, controlando al mismo tiempo los efectos sobre la composición de la fuerza de trabajo. Pero incluso en este contexto restrictivo, se producen nuevas contrataciones para cubrir vacantes de carácter temporal o para dotar de empleados públicos a nuevas organizaciones o programas. En tal caso, al comprobar las características de los nuevos empleados es importante determinar si el criterio de selección a seguir está en consonancia con los requisitos del puesto de trabajo a ocupar así como con las otras políticas de personal.

Finalmente, también se les exige a las propias unidades o servicios de personal una reducción significativa de sus dotaciones de plantilla ya que deben ser un ejemplo para las políticas que impulsan hacia el exterior y no, como a veces sucede, una excepción. La centralización de las políticas de personal es a menudo considerado como un mecanismo inhibidor de nuevas iniciativas o que limita la flexibilidad necesaria (KLIGNER, 1990; Lane 1994 ; Mesch, Perry y ReCaSino WISE, 1995) no permitiendo que las distintas áreas de gestión puedan impulsar sus propios programas y estrategias en materia de personal. Sin embargo, una consecuencia del aumento de la autonomía son las enormes diferencias en el coste de la estructura del personal de las distintas unidades administrativas o en los perfiles de los puestos de trabajo de aquellos que se encargan de la gestión del personal en los distintos servicios.

\begin{tabular}{|l|l|}
\hline \multicolumn{2}{|c|}{ Cuadro 2} \\
\multicolumn{1}{|c|}{ Pautas de crecimiento y de transformación Consejerias } \\
de las
\end{tabular}

Fuente: Elaboración propia. 
El Cuadro 2 muestra las medidas e indicadores a tener presentes para informar con respecto al crecimiento y a las características generales de los trabajadores que entran o salen de la Administración.

\section{ABSENTISMO}

El absentismo es en sí mismo un importante indicador de eficacia de una organización. En este sistema, se considera un indicador crítico dado el coste directo que conlleva un porcentaje elevado de absentismo y las negativas consecuencias que origina en la opinión pública, contribuyendo a asociar el sector público con la imagen peyorativa de la burocracia donde la mala administración conduce al fraude, a los abusos y a malgastar. Controlar el absentismo con precisión es el primer paso para solucionar el problema ya que esto demuestra un interés por parte de los dirigentes de la Administración pública y transmite la idea de que tomarse días libres sin un motivo justificado no se puede aceptar (DALTON y ENZ, 1988). Una política exitosa contra el absentismo requiere otros elementos discutidos por la literatura especializada -desde remodelar los puestos de trabajo a la acción disciplinaria- (MOORE, 1988), pero muchos de estas variables dependen de que previamente se tenga una buena idea de cual es la situación actual del absentismo, lo cual no siempre es fácil.

En el caso de Cataluña, únicamente se pudieron recoger datos sobre el absentismo por causas de enfermedad o de maternidad (ILT: Incapacidad Laboral Transitoria). Ambas son formas legales de absentismo, reguladas y controladas. Los responsables de la Dirección General de la Función Pública estaban interesados en recoger información en las diferentes Consejerías con respecto al número de personas-días perdidos y a conocer las características de los absentistas. Sin embargo, éstos son modos de absentismo que muy difícilmente pueden reducirse. Desde un punto de vista de gestión, sería mucho más interesante poseer datos sobre otros tipos de ausencias en el trabajo que pueden ser más evitables tales como los días que el personal se toma libres porque no se encuentran bien pero que no exigen un comprobante de enfermedad - según la legislación española - si un trabajador enfermo tarda menos de dos días en recuperarse ${ }^{3}$. Este y otros casos de ausencia no justificada no pueden ser controlados ya que las unidades de línea no siempre comprueban los días que los empleados no acuden a trabajar, ni tampoco lo hacen los servicios de personal de las Consejerías o los servicios de gestión financiera donde no se elaboran informes de cada empleado con la situación de su nivel de absentismo. Las medidas e indicadores a considerar con respecto al absentismo de los empleados públicos se resumen en el cuadro 3.

\begin{tabular}{|l|c|}
\hline \multicolumn{2}{|c|}{ Cuadro ? } \\
\hline \multicolumn{2}{|c|}{ Absentismo, por Consejerias } \\
\hline \multicolumn{1}{|c|}{ Indicadores } & \multicolumn{1}{c|}{ Temas $R H$} \\
\hline $\begin{array}{l}\text { Dias perdidos por persona y año } \\
\text { Porcentaje de empleados necesarios } \\
\text { para compensar el absentismo } \\
\begin{array}{l}\text { Costo anual de empleados exiras } \\
\text { Dias perdidos por persona y aǹo por: } \\
\text { grupos, nivel profesional, contrato la- } \\
\text { boral, sexo y edad, directivos y otros }\end{array}\end{array}$ & Perfil del absentista. \\
\hline
\end{tabular}

Fuente: Elaboración propia.

\section{SELECCIÓN, MOVIIIDAD INTERNA Y PROMOCIÓN}

Las organizaciones públicas requieren tanto personal con una estrecha gama de habilidades técnicas como de personal con una amplia variedad de aptitudes y habilidades y que estén capacitados a adaptarse a los cambios en la producción y en el suministro de los servicios públicos. En los sistemas continentales de función pública, como el modelo español, se contrata a personas con perfiles generalistas y a personas con perfiles especializados - por profesiones- a través de una oposición y/o concurso externo abierto, organizado centralizadamente y que utiliza unos procedimientos formalizados. Una cuestión fundamental para los departamentos de personal es cómo conseguir una selección descentralizada procurando que los distintos directivos estén más implicados en los procesos de selección. Otro objetivo es conseguir un sistema de reclutamiento que permita reducir el promedio de tiempo que se emplea en la contratación de personal (LAWLER 1988; KuINGNER, 1990). El número de solicitantes y la necesidad de efectuar una criba de un modo más o menos rápido, económico y respetuoso con los valores públicos de mérito, igualdad de oportunidades e imparcialidad, constituyen una importante limitación en esta área.

El escaso control directo sobre el número de solicitudes podría ser una cuestión importante en un contexto de expansión económica en el que las oportunidades del sector privado aparecen como más atractivas. Muy a menudo, la pregunta que se hacen los departamentos de personal es si éstos deben confiar la obtención de candidatos a fuentes internas o externas, y si es posible equiparar las expectativas de las personas en busca de alternativas de trabajo dentro de la organización con el interés y la planificación estratégica de las unidades administrativas (SCHULER, 1988). Obviamente, todo dependerá en primer lugar, de lo que la organización ofrezca a la gente de dentro y fuera 
de la misma y del nivel de competencia que exista para tales puestos.

Para obtener una mejor comprensión de las pautas de movilidad intraorganizativa para un uso más eficaz de los recursos humanos (ABELSON, FERIS y URBAN, 1988), puede resultar útil controlar las pautas de movilidad que siguen los empleados públicos dentro de la Administración. Estas incluyen la promoción -movilidad vertical - y cambiar el puesto de trabajo de un departamento a otro - movilidad horizontal- También existe la movilidad geográfica — de la periferia al centro o viceversa-. En el caso de la Generalidad, se obtuvo la la impresión de que estos procesos están controlados más por los empleados individualmente que por la Administración en sí misma, en el sentido de que las personas anticipan las decisiones sobre la movilidad y si poseen las suficientes cualidades y tienen suerte, podrán tener éxito en su trabajo y avanzar gradualmente en las distintas categorías salariales establecidas dentro de cada grupo, con independencia de las necesidades objetivas de la Administración en cuanto a la distribución de su personal.

Con respecto a las carreras de los empleados que ocupan jefaturas administrativas, los candidatos pueden ser escogidos a nivel interno o seleccionados por fuentes externas. En una organización donde los candidatos son escogidos a nivel interno, tal como sucede en la administración catalana, esta política puede ser considerada como una gratificación para algunas personas. Sin embargo, esto obliga a la organización a facilitar formación y oportunidades de promocionar profesionalmente, así como un sistema abierto y justo en el que los candidatos potenciales puedan confiar. La competencia se reduciría si existiera un limitado grupo de candidatos potenciales o cuando la gente tiene el convencimiento de que el sistema no ofrece una igualdad de oportunidades para los candidatos.

La vinculación de la promoción a la asunción de jefaturas administrativas ha sido el motivo que ha impulsado la existencia de muchos puestos directivos (inflación orgánica) en los que en realidad no se ejercen funciones de dirección (los puestos de jefes de negociado suelen ser en muchas administraciones un ejemplo de este fenómeno). En los niveles inferiores de la escala de dirección, los supervisores de primera línea siguen manteniendo su posición jerárquica mientras que su labor directiva ha desaparecido mediante la automatización o ha sido absorbida por el progreso. Como consecuencia, los directivos que desean implantar sistemas organizativos avanzados - sobre la base de estructuras planas y equipos de trabajo básicos, sin contar con supervisores en el sentido tradicional-, se encuentran con dificultades para llevarlos a cabo. Otra consecuencia negativa de este sistema es que los empleados más profesionalizados con experiencia y deseos de promocionar se ven forzados a entrar en la competición para la promoción hacia puestos de dirección cuando en verdad preferirían conti- nuar haciendo su trabajo profesional o técnico. Los estudios sobre recursos humanos han discutido la oportunidad de escaleras múltiples de promoción profesional como un modo de ampliar las oportunidades de promoción para los trabajadores sin tener que asumir necesariamente responsabilidades directivas (DruCKer, 1986; SCHULER, 1988; Gore 1993). La ampliación de las posibilidades de promoción no limitada a un determinado conjunto de tareas es otra posibilidad que ofrece a los empleados públicos la oportunidad de adquirir nuevas habilidades en áreas funcionales diversas, si bien el timepo necesario para adquirir nuevas habilidades será necesariamente más largo. El cuadro 4 refleja un resumen de las medidas e indicadores utilizados para la producción de indicadores orientados a comprender mejor el proceso de selección, movilidad y promoción dentro de la organización.

\begin{tabular}{|c|c|}
\hline \multicolumn{2}{|c|}{$\begin{array}{l}\text { Cuadro } 4 \\
\text { Selección, movilidad interna y promoción por } \\
\text { Consejerias }\end{array}$} \\
\hline Indicadores & Temas RH \\
\hline $\begin{array}{l}\text { Puestos ofrecidos por unidades cuer- } \\
\text { pos generales vs. cuerpos especiales }\end{array}$ & $\begin{array}{l}\text { Peso relativo del personal de gestión } \\
\text { general/especialistas en los nuevos } \\
\text { puestos. }\end{array}$ \\
\hline $\begin{array}{l}\text { Porcentaje de puestos para plantilla } \\
\text { interna o candidatos externos }\end{array}$ & Origen de los nuevos empleados. \\
\hline Ratio de solicitantes/puestos a cubrir & Demanda para puestos públicos. \\
\hline Porcentaje de candidatos aprobados & Cualificaciones de los solicitantes. \\
\hline $\begin{array}{l}\text { Promedio de tiempo —días- para el } \\
\text { proceso de selección }\end{array}$ & $\begin{array}{l}\text { Tiempo empleado para cubrir las va- } \\
\text { cantes. }\end{array}$ \\
\hline $\begin{array}{l}\text { Recursos administrativos en los pro- } \\
\text { cesos de selección }\end{array}$ & Precisión en los procedimientos. \\
\hline $\begin{array}{l}\text { Composición de los comités de selec- } \\
\text { ción }\end{array}$ & $\begin{array}{l}\text { Implicación de las partes interesadas } \\
\text { en los procesos de selección. }\end{array}$ \\
\hline $\begin{array}{l}\text { Empleados que cambian sus puestos } \\
\text { por grupos, nivel de carrera, tipo de } \\
\text { contrato, sexo y edad, directivos y } \\
\text { otros }\end{array}$ & $\begin{array}{l}\text { Perfil de los empleados que cambian } \\
\text { sus puestos en la organización. }\end{array}$ \\
\hline $\begin{array}{l}\text { Ratio de cambios inter/intra Conse- } \\
\text { jerías }\end{array}$ & $\begin{array}{l}\text { Movilidad interna a las Consejerías o } \\
\text { entre distintas Consejerias. }\end{array}$ \\
\hline $\begin{array}{l}\text { Ratio de incorporaciones/salidas de } \\
\text { un departamento }\end{array}$ & $\begin{array}{l}\text { Consejerías que tienden a ganar/per- } \\
\text { der empleados. }\end{array}$ \\
\hline $\begin{array}{l}\text { Porcentaje de cambios verticales por } \\
\text { grupops, nivel de carrerd, lipo de con- } \\
\text { irato, directivos y otros. }\end{array}$ & Movilidad vertical. \\
\hline $\begin{array}{l}\text { Ratio de cambios dentro y fuera de la } \\
\text { capital }\end{array}$ & Movilidad geográfica. \\
\hline $\begin{array}{l}\text { Número de candidatos en los concur- } \\
\text { sos para puestos directivos }\end{array}$ & $\begin{array}{l}\text { Competición en los concursos de } \\
\text { méritos para puestos de directivos. }\end{array}$ \\
\hline
\end{tabular}

Fuente: Elaboración propia. 


\section{RETRIBUCION Y EVALUACIÓN DEL DESEMPEÑO}

$\mathrm{Al}$ igual que en muchas organizaciones públicas, los empleados de la Administración de la Generalidad tienen asignados un nivel de sueldo que es fundamentalmente fijo, sin tener en cuenta como se realiza el trabajo o el valor de este trabajo para la Administración. El sueldo tiene un componente variable que básicamente depende de la productividad y de la antigüedad en el trabajo. No obstante, el componente del sueldo asignado a la productividad es generalmente demasiado pequeño para generar diferencias significativas entre los empleados. La consecuencia directa es que no llega a ser un instrumento eficaz para motivar al empleado y, de esta manera, ayudar a alcanzar un nivel más alto de productividad. Más bien al contrario, está siendo utilizando de un modo negativo por algunos directivos, debido a la falta de unos criterios claros a la hora de asignar dichos complementos, lo cual repercute en quejas y conflictos dentro de la plantilla.

Dado que al personal se le paga un sueldo base dependiendo del puesto de trabajo que ocupa y que consta en una tabla retributiva general, la cuestión fundamental es si hay equidad interna en el sentido de que trabajos comparables tienen la misma posición en la estructura retributiva. Dada la dificultad para medir cuantitativamente la equidad interna, algunos otros indicadores pueden dar una percepción de la respuesta burocrática a la percepción de injusticia en la forma en que empleados individuales son retribuidos. Tres maneras de esquivar y superar la rigidez del sistema retributivo pasan por 1) modificar la catalogación de los puestos, 2) crear nuevos puestos directivos y 3) pagar horas extras. Estas tres medidas pueden tener consecuencias financieras y estructurales de gran importancia lo que refuerza la necesidad de mantenerlas bajo control.

La otra pregunta básica es cómo mejorar el sistema de mérito, es decir, que el sueldo dependa más del rendimiento individual, dando un mayor peso relativo al componente variable de la retribución (KANTER 1988). La literatura sobre retribuciones en el sector público es bastante crítica con los sistemas retributivos que premian el rendimiento individual ya que considera que generan pocas diferencias en los sueldos y los directivos continúan teniendo dificultades para medir los resultados en el trabajo, por lo que sugieren diferentes maneras de pagar a los empleados (PERRY, 1992). En el momento en que esta investigación se llevaba a cabo, la Dirección General de la Función Pública estaba experimentando con nuevos procedimientos de evaluación del rendimiento en el trabajo. Respecto a este programa en particular era importante controlar su cobertura, la forma de la distribución de la "notas" puestas a los empleados y el valor total de las recompensas. El cuadro 5 muestra un resumen de estas medidas e indicadores.

\begin{tabular}{|l|l|}
\hline \multicolumn{2}{|c|}{ Cuadro 5} \\
\multicolumn{2}{|c|}{ Retribuciones y evaluación del desempeño } \\
por Consejeria
\end{tabular}

Fuente: Elaboración propia.

\section{FORMACIÓN Y SOCIALIZACIÓN}

La formación es un elemento crítico en el ciclo de la Dirección y Gestión de Recursos Humanos ya que cualquier cambio en la composición de la función pública, en el absentismo, los sistemas de selección, los criterios de promoción, las remuneraciones y la evaluación del desempeño dependen hasta un cierto punto de la capacidad que tenga la organización de suministrar unas oportunidades de formación que sean adecuadas. Las administraciones públicas tienen tendencia a ser estables lo cual les permite formular planes de formación y desarrollar actividades de mejora de la capacitación que posibilitan alcanzar las necesidades de los empleados y de la organización a largo plazo. No obstante, la formación muchas veces está considerada también como un sistema para combatir el estrés y para mantener la plantilla motivada y leal a la organización. De esta manera la formación es percibida, más como un beneficio para el empleado que como una herramienta gerencial (OLSON, 1988). La cuestión fundamental para los departamentos de personal es, pues, si los Programas de Formación están sistemáticamente enlazados con los otros programas y actividades de la Dirección y Gestión de Recursos Humanos o bien sólo sirven para desarrollar o satisfacer los objetivos personales de los empleados.

La literatura sobre el capital humano (BECKER, 1964; THuROw, 1970) hace una distinción entre KSAs (conocimientos, técnicas y habilidades) que son comunes a muchos trabajos y empresarios y que configuran el perfil del trabajador que prefieren la mayoría de los directivos, de aquellas que son únicamente preferidas y valoradas por unos pocos directivos en función del puesto y de la organización.. La formación puede mejorar las 
KSAs del empleado necesarias para mejorar su rendimiento en sus tareas o puede ser ofrecida para capacitar el aprendizaje de KSAs que no tienen relación con el trabajo que ejecuta normalmente el empleado, y que pueden servir, por tanto, para otros desempeños. En la Administración de la Generalidad, la Escuela de Administración Pública de Cataluña suministra cursos generales mientras que las Consejerías y algunas unidades administrativas concretas organizan cursos que están más orientados a áreas especificas y a KSAs relacionadas con sus actividades. El apoyo que reciben los departamentos de formación o la Escuela de Administración Pública depende claramente de si logran obtener economías de escala o contribuyen a la difusión de cambios gerenciales, o de si juegan un papel importante en la configuración de una cultura organizativa que sea adecuada a la estrategia de la organización.

El primer paso en la evaluación de estas variables es el control de las actividades formativas, las características de los participantes y el coste por participante. El cuadro 6 ofrece un resumen de estas medidas. Los procesos de socialización también pueden ser desarrollados mediante los procesos formativos o por mecanismos más informales que operan en el puesto de trabajo y en su entorno. No obstante, estos elementos son más difíciles de identificar. Sus resultados en términos de su fijación psicológica y del compromiso individual con la organización (SCHEIN, 1992) pueden ser evaluados por medio de una encuesta utilizando un cuestionario estructurado para este objetivo.

\begin{tabular}{|c|c|}
\hline \multicolumn{2}{|c|}{$\begin{array}{l}\text { Cuadro } 6 \\
\text { Formación y Socialización } \\
\text { por Consejerias }\end{array}$} \\
\hline Indicadores & Temas RH \\
\hline $\begin{array}{l}\text { Horas de formación por persona y } \\
\text { tema }\end{array}$ & $\begin{array}{l}\text { Diferencias en el esfuerzo de forma- } \\
\text { ción entre las Consejerías. }\end{array}$ \\
\hline $\begin{array}{l}\text { Distribución de los empleados por gru- } \\
\text { pos y temas }\end{array}$ & $\begin{array}{l}\text { Adecuación de programas de forma- } \\
\text { ción a las necesidades de los emplea- } \\
\text { dos. }\end{array}$ \\
\hline $\begin{array}{l}\text { Trabajadores que han asistido a: } \\
1 \text { curso } \\
2-5 \text { cursos } \\
6-10 \text { cursos } \\
10+\text { cursos }\end{array}$ & $\begin{array}{l}\text { Cobertura de programas de formación. } \\
\text { Hasta qué punto la organización con- } \\
\text { trola los planes de formación de los } \\
\text { individuos. }\end{array}$ \\
\hline $\begin{array}{l}\text { Coste promedio por hora de forma- } \\
\text { ción }\end{array}$ & Coste de formación por Consejerias. \\
\hline
\end{tabular}

Fuente: Elaboración propia.

\section{RELACIONES LABORAIES}

Nuevos puntos de vista gerenciales implican un cambio del clima laboral. La idea básica es que los empleados tomen la responsabilidad por el trabajo que realizan, individualmente o en equipo, como una manera de contribuir al rendimiento de la organización. Para apoyar este proceso se requieren muchas transformaciones en la estructura de la organización, en las descripciones de los puestos de trabajo, en la formación, en la participación en los procesos de definición de objetivos, de los sistemas retributivos, y en otras políticas de personal. Idealmente nuevas formas de gestión deberían traducirse en organizaciones donde las diferencias de puntos de vista entre los empleados y la dirección son muy escasas, donde existen unos valores generales compartidos en todos los niveles de la organización y existe una confianza básica y una concordancia entre el interés del individuo y el de la institución. Pero, en realidad, una organización no puede eliminar las diferencias básicas entre los empleados y la dirección como ocurre en los conflictos colectivos y en la confrontación directa sobre derechos o intereses. Como ha sugerido BeER et al. (1985), los empleados tienen sus propios objetivos e intentaran mejorar su interés económico, satisfacción psicológica y sus derechos. De hecho los empleados que estén bien organizados pueden tener una influencia que puede beneficiar a la organización en su esfuerzo de equilibrar su objetivo de eficiencia y justicia. La habilidad de los directivos de personal para resolver sus problemas con los empleados y con los sindicatos es un buen indicador de su eficacia.

La representación sindical, los procesos de negociación y los procedimientos para resolver conflictos suelen permitir solventar las controversias de un modo pacífico. Toda vez que conocer los datos sobre la afiliación sindical - un buen indicador sobre las potencialidades del sistema de relaciones laborales de una organización-, es un tema delicado y controvertido, los indicadores y las medidas seleccionadas se centran en las características generales de los representantes de los sindicatos (grupo y cuerpo al que pertenecen, tipo de contrato) y en su distribución por Consejerías. El seguimiento de las huelgas convocadas y otras medidas que afectan al trabajo y que implican una pérdida de horas laborales son un indicador de la dificultad que existe entre la gestión de conflictos colectivos. Otro indicador que muestra el estado de las relaciones laborales son los recursos internos que presentan los empleados públicos contra decisiones o actuaciones de la Administración y que, en caso de que no se concilien las posiciones, los empleados pueden dar lugar a la presentación de demandas judiciales contra la Administración.

Otra fuente de conflictos individuales es la aplicación del régimen disciplinario. Las sanciones pueden ser necesarias para controlar el comportamiento de los empleados en el sentido más básico, cuando aparecen problemas de naturaleza diversa - desde llegar tarde a los puestos de trabajo o estar ausente sin permiso hasta actuaciones poco éticas. Las administraciones públicas tie- 
nen procedimientos muy formalizados para tratar estas situaciones que protegen los derechos de los empleados a los que se desea sancionar. No obstante, la mayoría de los jefes administrativos se sienten incómodos con estas situaciones y tienen la tendencia a evitar el inicio de un expediente sancionador e incluso la formulación de una advertencia por escrito. Lo más usual es que ante comportamientos irregulares de un empleado público su superior tenga, como mucho, una conversación con este empleado. En la Administración de la Generalidad una solución intermedia es la de no iniciar un expediente disciplinario y sólo formular una advertencia que no tiene validez formal pero sí simbólica. Este sistema fue muy difícil revisar ya que las advertencias eran borradas al transcurrir unos pocos dias. El resultado final es que poca o ninguna acción disciplinaria fue adoptada por los distintos directivos de línea salvo en los casos del cuerpo de policía, bomberos o personal penitenciario, donde la disciplina es fundamental y está muy valorada. Para los departamentos de personal el asunto es si la acción disciplinaria esta siendo bien utilizada, mal utilizada o se abusa de ella. Algunos sencillos indicadores en el área de relaciones laborales nos pueden dar una indicación como se demuestra en el cuadro 7.

\section{Conclusiones}

En este artículo se presenta un sistema de indicadores para el control de la gestión de los recursos humanos en base a una aplicación desarrollada para la Administración de la Generalidad de Cataluña. Durante el diseño y la implementación del sistema ya se consideró que esta metodología podía ser aplicada en otros contextos ya que los problemas tratados son comunes en todos los programas de Dirección y Gestión de Recursos Humanos. También se percibió que el éxito final de la metodología depende más del apoyo político y de la capacidad de persuasión de los directivos impulsores del programa que de las dificultades técnicas en cuanto a la recogida de datos, la elaboración de los indicadores y la configuración de un informe integrado. La resistencia demostrada por algunas Consejerías a facilitar la información sólo pudo ser superada con el apoyo y la implicación de algunos cargos de alto nivel.

En general, la aplicación del sistema reveló que fue un instrumento útil para hacer el cambio hacia nuevos modelos de gestión ya que la implantación del sistema facilitó la información necesaria a los responsables de la Dirección General de la Función Pública sobre el éxito de sus iniciativas. La información recogida les servió, por tanto, para discutir, corregir y mejorar sus propias estrategias. Además, el sistema permite el benchmarking dentro de la organización - al poder comparar datos entre las Consejerías - o bien, con otras administraciones

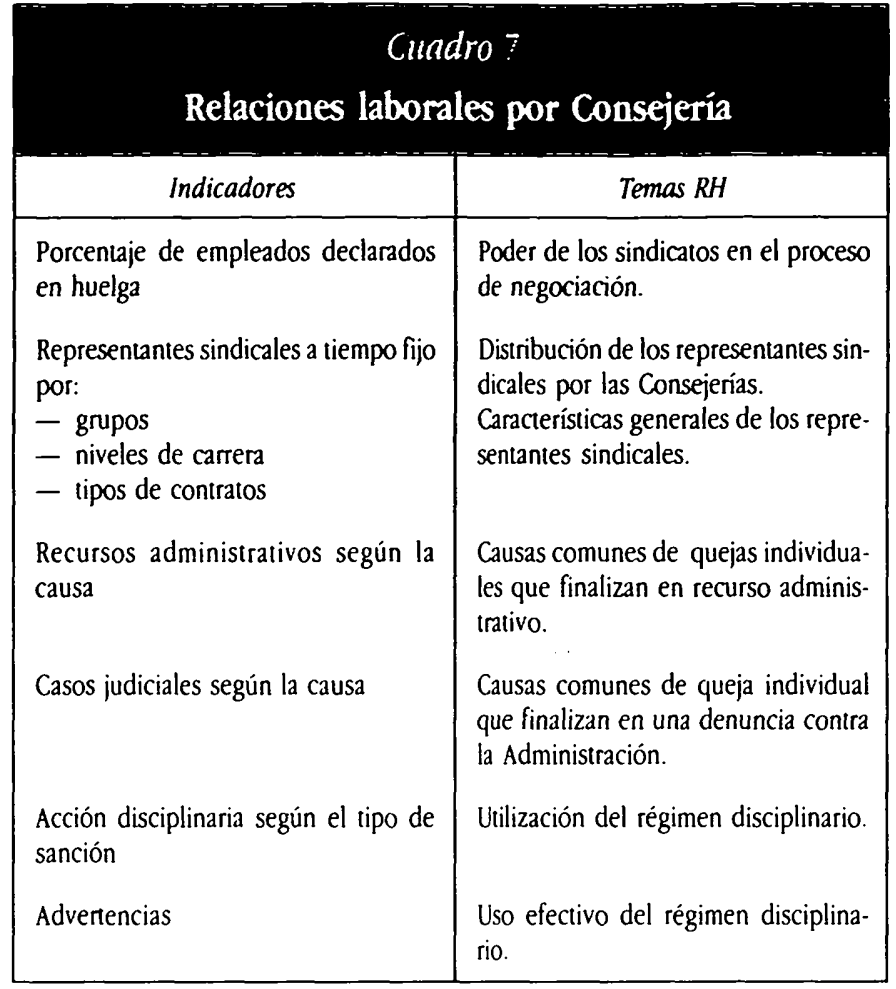

Fuente: Elaboración propia.

públicas cuando éstas dispongan de datos similares. Si se distribuye esta información por las distintas unidades de la organización se incentiva el interés en el proyecto al comprobar su utilidad, además de facilitar una base informativa para que otros directivos de línea vean algunos temas de per-sonal como factores críticos para la estrategia de sus departamentos.

El sistema podría ser utilizado con un objetivo básico de control. Sin embargo, más bien fue utilizado para dar, a los responsables administrativos tanto de la Dirección General de la Función Pública como de los servicios de personal de las Consejerías, una visión integrada y algún sentido sobre lo que se podía conseguir en las diferentes áreas de la Dirección y Gestión de Recursos Humanos. Empujar a los miembros de la organización hacia objetivos que parecían razonables fue no sólo bien recibido sino gratamente aceptado, dado que existía un sentimiento de que esto conllevaba un aprendizaje y se estaba ejerciendo un liderazgo positivo para la organización. La Dirección General de la Función Pública podía ofrecer algo que se consideraba de valor para los distintos encargados de la gestión de personal desperdigados por la Administración. La propuesta efectuada por la Dirección General de la Función Pública encajaba perfectamente con la filosofía del equipo de investigación que se basa en asumir que movilizar día a día la administración de personal hacia una Dirección y Gestión de Recursos Humanos más estratégica requiere, en primer lugar, un buen conocimiento del actual rendimiento con respecto a los factores críticos de la política de personal. 
- Universidad Autónoma de Barcelona.

* Universidad Pompeu y Fabra.

1 La Generalidad de Cataluña posee unos 110.000 empleados públicos, pero si se excluyen los colectivos de personal docente y de personal estatutario (empleados del sistema sanitario) la cifra total que se alcanza es de 30.000 . Estos $30.000 \mathrm{em}$ pleados, que son el objeto del presente estudio, representan el núcleo de la función pública catalana, incluyendo colectivos especiales como la policía autonómi$\mathrm{ca}$, los bomberos, el personal penitenciario, los agentes rurales, etc. La Administración de la Generalidad de Cataluña estaba organizada, en el momento de la realización del estudio, en 14 Consejerias (actualmente hay 13). Cada Consejeria posee una
Secretaria general que integra los servicios comunes del departamento (en donde están ubicados los servicios de personal) y varias Direcciones Generales. La Dirección General de la Función Pública es la unidad central de gestión del personal de la Generalidad y está ubicada en la Consejería de la Presidencia.

${ }^{2}$ Los miembros del equipo que realizaron este estudio, además de los autores del articulo, son: Ioan Subirats (direcior del proyecto), Miquel Salvador y Lluís FerRer. ${ }^{3}$ Muchas entidades tienen un límite en el número de días libres a ausentarse en el trabajo que no requieren la presentación de baja por enfermedad o para asuntos personales. Lo que lleva a utilizar estos días en beneficio propio.

\section{Bibliografía}

Abelson, M., FerRis, G. and Urban, T. (1988): "Human resource Development and Employee Mobility", en SCHulfr, R., YoungBtood, S. and HubFr, V. (eds.) Readings in Personnel and Human Resource Management. St. Paul, MN, West Publishing.

Barzelay, M. (1992): Breaking Through Bureaucracy. Berkeley, University of California Press.

Befr, M., Spector, B., Lawrence, P., Mills, Q., Walton, R. (1985): Human Resource Management. New York, The Free Press.

BECKER, G. (1964): Human Capital: A Theoretical and Empirical Analysis with Special Reference to Education. New York: National Bureau of Economic Research.

DalToN, D., ENZ, C. (1988): "New Directions in the Management of Employee Absenteeism: Attention to Policy and Culture", en SChuler, R., Youngbloon, S. and HUBER, V. (eds.) Readings in Personnel and Human Resource Management. St. Paul, MN, West Publishing.

Donahue, J. (1989): The Privatization Decision. New York, Basic Books.

DruCKER, P. (1986): "Good-bye to the Old Personnel Department", Wall Street Journal, May 22.

GORE, A. (1993): The Gore Report on Reinventing Government. New York, Random House.

Hood, C. (1991): "A Public Management for All Seasons?", Public Administration 69, (spring), pp. 3-19.

KanTER, R. (1988): "From Status to Contribution: Some Organizational Implications of the Changing Basis for Pay", en Foulkes, F. (ed.) Human Resources Management: Readings. Englewood Clifss, NJ: Prentice Hall.

Kelman, S. (1990): "The Renewal of the Public Sector", American Prospect, 2 (summer), pp. $51-57$.

KuIGner, D. (1990): "Variables Affecting the Design of State and Local Personnel Systems", en Heys, S. and Kearney, R. (eds.) Public Personnel Administration. Englewood Cliffs, NJ.Prentice Hall.

Lane, L. (1994): "Public Sector Performance Management", Review of Public Personnel Administration, XIV, 3: 26-44

LAWLER, E. (1988): "Human Resources Management: Meeting the New Challenges", en Foulkfs, F. (ed.) Human Resources Management: Readings. Englewood Clifss, NJ: Prentice Hall.

MCGregor Jr., E. (1988): "The Public Sector Human Resource Puzzle: Strategic Management of a Strategic Resource", Public Administration Review, 48 (novemberdecember): $941-950$.

MesCH, D., PerRY, J., ReCasino W/SE, L. (1995): "Bureaucratic and Strategic Human resource Management: An Empirical Comparison in the Federal Government", Journal of Public Administration Research and Theory, 5: 385-402.

Milfs, R., SNow, C. (1984): "Designing Strategic Human Resource Systems." Organizational Dynamics 12: 36-51.

Moore M. L. (1988): "Components of Absenteeism Control Programs", en Schulf.R, R., YOUNGBLOOD, S. and HUBER, V. (eds.) Readings in Personnel and Human Resource Management. St. Paul, MN, West Publishing.

Moore, M. H. (1995): Creating Public Value. Cambridge, Ma. Harvard University Press.

OLSON, L. (1988): "Training Trends: The Corporate View", en SCHuler, R., YOungBLoOD, S.and HUBER, V. (eds.) Readings in Personnel and Human Resource Management. St. Paul MN, West Publishing.

Perry, J. (1992): "The Merit Pay Reforms", en Ingraham, P. and Rosenblom, D. (eds.) The Promise and Paradox of Civil Service Reform, Pittsburgh: University of Pittsburg Press. pp. 199-215.

- (1993): "Strategic Human Resource Management: Transforming Federal Civil Service." Review of Public Personnel Administration, 13, 4:59-71.

SCHEIN, E. (1992): Organizalional Culture and Leadersbip. San Francisco: JosseyBass.

SChuler, R. (1988): "Human Resource Management Practice Choices", en Scruler, R., YoungBLood, S.and HuBer, V. (eds.) Readings in Personnel and Human Resource Management. St. Paul MN, West publishing.

SChuler, R. and MaCMillan, I. (1984): "Gaining Competitive Advantage Through Human Resource Management Practices", Human Resource Management, 23, 3, pp 241-255.

Thurow, L. (1970): Investment in Human Capital. Belmont, CA: Wadsworth. 